\title{
Neutrino Trapping and Accretion Models for Gamma\#Ray Bursts
}

\section{Citation}

Di Matteo, Tiziana, Rosalba Perna, and Ramesh Narayan. 2002. “Neutrino Trapping and Accretion Models for Gamma\#Ray Bursts." The Astrophysical Journal 579 (2): 706-15. https:// doi.org/10.1086/342832.

\section{Permanent link}

http://nrs.harvard.edu/urn-3:HUL.InstRepos:41384941

\section{Terms of Use}

This article was downloaded from Harvard University's DASH repository, and is made available under the terms and conditions applicable to Other Posted Material, as set forth at http:// nrs.harvard.edu/urn-3:HUL.InstRepos:dash.current.terms-of-use\#LAA

\section{Share Your Story}

The Harvard community has made this article openly available.

Please share how this access benefits you. Submit a story.

Accessibility 


\title{
Neutrino Trapping and accretion models for Gamma-ray Bursts
}

\author{
Tiziana Di Matteo ${ }^{1,2}$, Rosalba Perna ${ }^{3,4}$, Ramesh Narayan ${ }^{3,6}$
}

\begin{abstract}
Many models of Gamma Ray Bursts (GRBs) invoke a central engine consisting of a black hole of a few solar masses accreting matter from a disk at a rate of a fraction to a few solar masses per second. Popham et al. and Narayan et al. have shown that, for $\dot{M} \gtrsim 0.1 \mathrm{M}_{\odot} \mathrm{s}^{-1}$, accretion proceeds via neutrino cooling and neutrinos can carry away a significant amount of energy from the inner regions of the disks. We improve on these calculations by including a simple prescription for neutrino transfer and neutrino opacities in such regions. We find that the flows become optically thick to neutrinos inside a radius $R \sim 6-40 R_{\mathrm{s}}$ for $\dot{M}$ in the range of $0.1-10 \mathrm{M}_{\odot} \mathrm{s}^{-1}$, where $R_{\mathrm{s}}$ is the black hole Schwarzchild radius. Most of the neutrino emission comes from outside this region and, the neutrino luminosity stays roughly constant at a value $L_{\nu} \sim 10^{53} \mathrm{erg} \mathrm{s}^{-1}$. We show that, for $\dot{M} \gtrsim 1 \mathrm{M}_{\odot} \mathrm{s}^{-1}$, neutrinos are sufficiently trapped that energy advection becomes the dominant cooling mechanism in the flow. These results imply that $\nu \bar{\nu}$ annihilation in hyperaccreting black holes is an inefficient mechanism for liberating large amounts of energy. Extraction of rotational energy by magnetic processes remains the most viable mechanism.
\end{abstract}

Subject headings: accretion, accretion disks — black hole physics — gamma rays: bursts - radiation mechanisms: thermal

\section{Introduction}

Most popular models of Gamma Ray Bursts (GRBs) invoke a binary merger or a collapse involving compact objects. In particular, these include mergers of double neutron star binaries (Eichler et al. 1989; Narayan, Paczynski \& Piran 1992; Ruffert \& Janka 1999), mergers of a neutron star with a black hole (Paczynski 1991; Narayan et al. 1992; Ruffert \& Janka 2001 and

\footnotetext{
${ }^{1}$ Max-Planck-Institute für Astrophysik, Karl-Schwarzschild-Str. 1, 85740 Garching bei München, Germany

${ }^{2}$ Carnegie-Mellon University, Dept. of Physics, 5000 Forbes Ave., Pittsburgh 15231

${ }^{3}$ Harvard-Smithsonian Center for Astrophysics, 60 Garden St., Cambridge, MA 02138

${ }^{4}$ Harvard Society of Fellows, 78 Mt Auburn Street, Cambridge, MA 02138

${ }^{6}$ Institute for Advanced Study, School of Natural Sciences, Einstein Drive, Princeton, NJ 08540
} 
references therein), of helium star with a black hole (Fryer \& Woosley 1998), "collapsars" or "failed supernovae" (Woosley 1993; Paczynski, 1998; MacFadyen \& Woosley 1999; MacFadyen, Woosley \& Heger 2001) and "supranovae" (Vietri \& Stella 1998). All of the above scenarios lead to the formation of a black hole with a debris torus or disk around it. (The only exceptions are models in which the GRB energy is provided by the magnetic and rotational energy of the newly formed neutron star; e.g.; Usov 1992). In order to understand how the extraordinary amount of energy characteristic of GRBs can be extracted, we are motivated to further examine the properties of such compact and massive disks around black holes.

Accretion models in the context of GRBs have been recently discussed by Popham, Woosley \& Fryer (1999; hereafter PWF), Narayan, Piran \& Kumar (2001; hereafter NPK) and Kohri \& Mineshige (2002). The typical mass accretion rates in GRB models are extremely high, of the order of a fraction of solar mass up to a few solar masses per second. Under such conditions, the gas photon opacities are also very high and radiation becomes trapped (see e.g.; Katz 1977; Begelman 1978; Abramowicz et al. 1988). However, at sufficiently high mass accretion rates, although energy advection remains important in the outer parts, the disk becomes dense and hot enough in the inner regions to cool via neutrino emission. For this reason PWF named these disks neutrino-dominated accretion flows (NDAFs). This regime is of particular interest, because neutrinos can, in principle (see e.g. NPK; Ruffert \& Janka 1999), tap the thermal energy of the disk produced by viscous dissipation and liberate large amounts of its binding energy (via the $\nu \bar{\nu} \rightarrow e^{+} e^{-}$process in regions of low baryon density). For this mechanism to be efficient, though, the neutrinos must escape before being advected into the black hole.

In this paper we investigate the effects of neutrino transport within the context of NDAFs. By using a simple prescription to account for neutrino scattering and absorptive opacities, we find that, for accretion rates $\dot{M} \gtrsim 1 \mathrm{M}_{\odot} \mathrm{s}^{-1}$, the gas becomes increasingly more opaque and neutrinos become trapped. As a result, energy advection becomes the dominant cooling mechanism in the inner regions of the flows. We show that, as $\dot{M}$ increases, the disk emitting surface moves further out in radius and the neutrino luminosity of the flow remains nearly constant. We show that the accretion flow luminosity plateaus as it approaches the neutrino Eddington limit of the inner disk and as the neutrino cooling efficiency decreases.

NPK and PWF also noted the importance of neutrino opacity at $\dot{M} \gtrsim 1 \mathrm{M}_{\odot} \mathrm{s}^{-1}$ but did not allow for neutrino transport effects in their models. Our work therefore complements the earlier studies of NDAFs. A detailed treatment of neutrino transfer was included in the numerical simulations carried out by Ruffert \& Janka (1999), where accretion tori are formed as a result of neutron star merging. Consistent with our findings, these authors also show that opacities can be high in such tori. However, their results are specific to the parameter space covered by the merger model.

In $\S 2$ we identify the dominant neutrino opacity sources and outline the basic equations we use and the approximations we make to the neutrino transfer problem. In $\S 3$ we present our numerical 
results. We delineate the regions of parameter space in accretion rate and radius where the flow becomes optically thick to neutrino emission. In $\S 4$ we discuss the stability of the accretion flow. Finally, in $\S 5$, we compute the flow luminosity of our model, compare it with the derived neutrino Eddington limit and discuss various implications of our results for GRB energetics.

\section{The physical model}

\subsection{Neutrino emission and photodisintegration}

PWF and NPK have calculated models corresponding to steady-state accretion around a black hole with a high mass accretion rate. Their work showed that for the full range of accretion rates of interest $\left(0.01-10 \mathrm{M}_{\odot} \mathrm{s}^{-1}\right.$ ), and outside a certain radius $R \sim 100 R_{\mathrm{S}}$ (where $R_{\mathrm{S}}=2 G M / c^{2}=8.85 \times 10^{5} \mathrm{~m}_{3} \mathrm{~cm}$ is the Schwarzchild radius of a relativistic compact object of mass $M=3 m_{3} \mathrm{M}_{\odot}$ ), the disk is advection dominated. Inside this radius, and for $\dot{M} \gtrsim 0.1 \mathrm{M}_{\odot} \mathrm{s}^{-1}$, the temperature and density of the gas become high enough that neutrino cooling takes over fairly abruptly.

In such accretion flows neutrinos are generated both by neutronization and by thermal emission. The most significant thermal processes are:

(1) Electron-positron pair annihilation $\left(e^{-}+e^{+} \longrightarrow \nu_{i}+\bar{\nu}_{i}\right.$ where $i$ represents both electron-type neutrinos, $\nu_{e}-\bar{\nu}_{e}$, and heavy-lepton neutrinos $\left.\nu_{\mu}, \bar{\nu}_{\mu}, \nu_{\tau}, \bar{\nu}_{\tau}\right)$. The neutrino cooling rate per unit volume due to electron-positron pair annihilation is roughly given by (we take the PWF approximation to the Itoh et al. 1989; 1990 results):

$$
q_{\nu_{i}, \bar{\nu}_{i}}^{-} \simeq 5 \times 10^{33} T_{11}^{9} \quad \operatorname{erg~cm}^{-3} \mathrm{~s}^{-1}
$$

where $T_{11}=T / 10^{11} \mathrm{~K}$. We assume an equilibrium mixture of $e, \mu$ and $\tau$ neutrinos and antineutrinos so that the total cooling rate, when all species are included, is simply obtained by multiplying equation (1) by $N_{\nu}=3$, the number of neutrino flavors.

(2) Nucleon-nucleon bremsstrahlung $\left(n+n \longrightarrow n+n+\nu_{i}+\bar{\nu}_{i}\right)$. Bremsstrahlung cooling is represented by (Hannestad \& Raffelt 1998; Kohri \& Mineshige 2002):

$$
q_{\mathrm{brem}}^{-} \simeq 10^{27} \rho_{10}^{2} T_{11}^{5} .5 \quad \mathrm{erg} \mathrm{cm}^{-3} \mathrm{~s}^{-1},
$$

where $\rho_{10}$ is the scaled density, $\rho_{10}=\rho / 10^{10} \mathrm{~g} \mathrm{~cm}^{-3}$.

(3) Plasmon decay. This is the decay rate of the transverse plasmons, which are normal photons interacting with the electron gas through $\tilde{\gamma} \longrightarrow \nu_{e}+\bar{\nu}_{e}$, and is estimated by Ruffert,

\footnotetext{
${ }^{6}$ Thermal equilibrium is quickly established (in $\Delta t \lesssim 1 \mathrm{~ms}$ ) between the neutrinos and the matter via weak, neutral current annihilation of $e^{+} e^{-}$pairs (e.g.; Bethe, Applegate and Brown 1980; Salpeter \& Shapiro 1981).
} 
Janka \& Schäfer (1996) to be

$$
q_{\text {plasmon }}^{-} \simeq 1.5 \times 10^{32} T_{11}^{9} \gamma_{p}^{6} \exp ^{-\gamma_{p}}\left(1+\gamma_{p}\right)\left(2+\frac{\gamma_{p}^{2}}{1+\gamma_{p}}\right) \quad \operatorname{erg~cm}^{-3} \mathrm{~s}^{-1},
$$

where $\gamma_{p}=5.565 \times 10^{-2} \sqrt{\left(\pi^{2}+\eta_{e}^{2}\right) / 3}$ and $\eta_{e}=\mu_{e} / k T$, and we solve for $\mu_{e}$, the electron chemical potential, using equation (24) in Kohri \& Mineshige (2002).

The second type of neutrino cooling is due to neutronization reactions. The most significant of these in this context is electron-positron pair capture on nuclei $\left(p+e^{-} \longrightarrow n+\nu_{e} ; \quad n+e^{+} \longrightarrow \bar{\nu}_{e}+p\right)$, also know as the URCA process. The cooling rate per unit volume is given by (see also PWF):

$$
q_{e N}^{-}=q_{e^{-} p}^{-}+q_{e^{+} n}^{-} \simeq 9.0 \times 10^{33} \rho_{10} T_{11}^{6} X_{\text {nuc }} \quad \operatorname{erg~cm}^{-3} \mathrm{~s}^{-1},
$$

where $X_{\text {nuc }}$ is the mass fraction of free nucleons approximately given by (e.g.; PWF; Qian \& Woosley 1996):

$$
X_{\text {nuc }} \approx 34.8 \rho_{10}^{-3 / 4} T_{11}^{9 / 8} \exp \left(-0.61 / T_{11}\right),
$$

with an upper bound of unity. This prescription takes into account the transition from nucleon to $\alpha$-particles which effectively shuts off the URCA process. $X_{\text {nuc }}$ is typically very close to zero in the outer disk but photodisintegration breaks down $\alpha$ particles in neutrons and protons once $T$ reaches about $10^{10} \mathrm{~K}$. The photodisintegration process is also responsible for cooling the gas according to (PWF):

$$
q_{\text {photo }}^{-} \simeq 10^{29} \rho_{10} v \frac{d X}{d r} \quad \operatorname{erg~cm}^{-3} \mathrm{~s}^{-1},
$$

where $v$ is the disk radial velocity defined in $\S 2.3$.

At low optical depths the emission of neutrinos can be computed directly from the rates given in equations (1), (2), (3) and (4). NPK and PWF take into account neutrino cooling as described in equations (1) and (4) and follow the optically-thin approach in their computations.

\subsection{Neutrino opacities}

Each neutrino emission process, equations (1)-(4), has an inverse process corresponding to absorption. In addition, scattering impedes the free escape of neutrinos from the disk. The optical depths for neutrinos are therefore given by the inverse of the processes listed above plus a contribution from neutral-current scatterings off nucleons.

The inverse process to equation (1), i.e. the interaction of neutrinos with one another, gives rise to an optical depth:

$$
\tau_{a, \nu_{i} \bar{\nu}_{i}} \approx \frac{q_{\nu_{i}, \bar{\nu}_{i}}^{-} H}{4(7 / 8) \sigma T^{4}} \approx 2.5 \times 10^{-7} T_{11}^{5} H
$$


where the scale height $H$ is defined in $\S 2.3$. The term $(7 / 8) \sigma T^{4}$ is the Fermi-Dirac blackbody luminosity and is the same for each neutrino flavor, $\nu_{2}$ ]; $\sigma$ is the Stefan-Boltzmann constant.

The inverse process to equation (4), absorption onto protons or onto neutrons, leads to a neutrino absorptive optical depth in the flow given by (see also NPK),

$$
\tau_{a, e N} \approx \frac{q_{e N}^{-} H}{4(7 / 8) \sigma T^{4}} \approx 4.5 \times 10^{-7} T_{11}^{2} X_{\text {nuc }} \rho_{10} H
$$

Similarly, the inverse of bremsstrahlung and plasmon processes (given by Eqs. (2) and (3)) lead to two additional sources of opacity, $\tau_{a \text {,brem }}, \tau_{a, \text { plasmon, }}$, which can be worked out in the same way as for the previous two. In accordance with NPK and PWF, we find that electron-positron pair annihilation and electron pair capture on nuclei are always the dominant emission mechanism. Absorption via the inverse of equations (2) and (3) gives rise to much smaller absorptive optical depths than from equations (7) and (8). Although all absorption opacity sources are included in our code, we will mainly discuss those derived above.

A far more important opacity source (particularly for heavy-lepton neutrinos) comes from neutral-scattering off nucleons $\left(\nu_{i}+\{n, p\} \longrightarrow \nu_{i}+\{n, p\}\right)$. The cross section for momentum transfer for neutrino-nucleon scattering is given by

$$
\sigma_{s, N}=C_{s, N} \sigma_{0}\left(\frac{E_{\nu}}{m_{e} c^{2}}\right)^{2}
$$

where $E$ is the center of mass neutrino energy, $m_{e}$ is the electron rest mass, $c$ the speed of light, $\sigma_{0}=1.76 \times 10^{-44} \mathrm{~cm}^{2}$, and $C_{s, n}=\left(1+5 \alpha^{2}\right) / 24$ for neutrino-neutron scattering and $C_{s, p}=\left[4\left(C_{V}-1\right)^{2}+5 \alpha^{2}\right] / 24$ for neutrino-proton scattering, with $C_{V}=1 / 2+2 \sin ^{2} \theta_{W}$, $\sin ^{2} \theta_{W} \approx 0.23$ and $\alpha=1.25$ (e.g.; Shapiro \& Teukolsky 1983). We calculate the Rosseland mean opacity $\kappa_{s, N}$, by averaging the neutrino energy over the Fermi-Dirac distribution function with zero chemical potential. Using

$$
\left\langle\frac{1}{E_{\nu}^{2}}\right\rangle=\frac{5 /\left(7 \pi^{2}\right)}{(k T)^{2}}=\frac{1}{13.8(k T)^{2}}
$$

where $k$ is the gas Boltzmann constant, we find that the total opacity due to neutrino scattering is $\kappa_{s, N}=\kappa_{s, n}+\kappa_{s, p}=13.8\left(C_{s, p} Y_{p}+C_{s, n} Y_{n}\right) \sigma_{0} \rho\left(k T / m_{e} c^{2}\right)^{2}$, where $Y_{n}$ and $Y_{p}$ are the fractions of free neutrons $n$ and protons $p$, respectively. For completely dissociated matter, where this process is relevant, the nucleon mass fractions are $Y_{n}=1-Y_{e}$ and $Y_{p}=Y_{e}$. Here, we take $Y_{e} \sim 0.5$. This leads to a total scattering optical depth given by

$$
\tau_{s, \nu_{i}}=\rho \kappa_{s, N} H=2.7 \times 10^{-7} T_{11}^{2} \rho_{10} H
$$

\footnotetext{
${ }^{7}$ Note that there is a factor $1 / 2$ difference between electron-positron pairs and the neutrino-antineutrino contribution, which comes from comparing the electron spin degeneracy ( 2 spin states) to the neutrino spin degeneracy (one helicity state).
} 
which has the same temperature and density dependency as equation (8) (when $X_{\text {nuc }}=1$ ). Both PWF and NPK found that, for accretion rates above $\dot{m} \gtrsim 1$, where $\dot{m}=\dot{M} /\left(\mathrm{M}_{\odot} \mathrm{s}^{-1}\right)$, the disk becomes optically thick to its own neutrino emission. This typically occurs within the inner regions of the disk. However, neither PWF or NPK studied the effects of including, self-consistently in the models, the neutrino opacity sources described above. Here, we use a simple prescription to include neutrino transport and discuss its effects on models of NDAFs.

\subsection{Basic assumptions and equations}

In this study, (see also NPK), we adopt basic equations based on Newtonian dynamics. As in previous work (PWF and NPK), we are interested in the gross properties of the disks and in particular on the effects of neutrino transport. For simplicity, we use a steady state disk model. Even though accretion in a GRB engine is time dependent, the steady state assumption should be a reasonable approximation since the viscous timescales in the inner disk, where all the neutrinos processes become important, are much shorter than those in the outer disk, where $\dot{M}$ is expected to vary.

As in the standard theory of thin accretion disks (e.g., Shakura \& Sunyaev 1973; Frank, King \& Raine 1992), we consider height-averaged quantities and write the isothermal sound speed as $c_{s}^{2}=P / \rho$ and the vertical scale height as $H=c_{s} / \Omega_{K}$, where $\Omega_{K}=\left(G M / R^{3}\right)^{1 / 2}=2.4 \times 10^{4} m_{3}^{-1} r^{-3 / 2} \mathrm{~s}^{-1}$ is the Keplerian velocity and $r$ is in units of $R_{s}$. We adopt the standard Shakura-Sunyaev prescription for the kinematic viscosity coefficient, $\nu=\alpha c_{s}^{2} / \Omega_{K}$, and scale the dimensionless parameter $\alpha$ as $\alpha_{-1}=\alpha / 0.1$ (PWF, NPK). The continuity equation and an approximate expression for angular momentum balance (e.g., NPK) give the following relation for the mass accretion rate:

$$
\dot{M}=4 \pi R \rho H v \approx 6 \pi \nu \rho H .
$$

Correspondingly, the radial velocity of the gas is $v=3 \nu / 2 R$.

In the equation of state we include the contributions from radiation pressure, gas pressure, degeneracy pressure and neutrino pressure (see also Popham \& Narayan 1995; PWF and NPK).

$$
P=\frac{11}{12} a T^{4}+\frac{\rho k T}{m_{p}}\left(\frac{1+3 X_{\mathrm{nuc}}}{4}\right)+\frac{2 \pi h c}{3}\left(\frac{3}{8 \pi m_{p}}\right)^{4 / 3}\left(\frac{\rho}{\mu_{e}}\right)^{4 / 3}+\frac{u_{\nu}}{3},
$$

where $a$ is the radiation constant and the factor $11 / 12$ includes the contribution of relativistic electron positron pairs (as we expect the temperature to be significantly above the pair production threshold). In the degeneracy pressure term, $\mu_{e}$ is the mass per electron which we take to be equal to 2 in agreement with NPK and PWF. Neutrinos also contribute to the equation of state and this is taken into account in the forth term, where $u_{\nu}$ is the neutrino energy density defined as $u_{\nu}=7 / 8 a T^{4} \sum\left(\tau_{\nu_{i}} / 2+1 / \sqrt{3}\right) /\left(\tau_{\nu_{i}} / 2+1 / \sqrt{3}+1 / 3 \tau_{a, \nu_{i}}\right)$ (Popham \& Narayan 1995; see also eq. (15)). 
We write the energy equation as

$$
q^{+}=\frac{3 G M \dot{M}}{8 \pi R^{3}}=q^{-}+q_{\mathrm{adv}}
$$

where $q^{+}$represents the viscous dissipation, $q^{-}=q_{\nu}^{-}+q_{\mathrm{photo}}^{-} H$ is the total cooling rate due to both neutrino losses $\left(q_{\nu}^{-}\right)$and photodisintegration $\left(q_{\text {photo }}\right.$, Eq. 6$)$ and $q_{\text {adv }}$ is the advective cooling rate. All the $q$ 's correspond to half the disk thickness. For the neutrino flux $q_{\nu}^{-}$, we use a simplified model for the transport, based on the two-stream approximation and derived by Popham \& Narayan (1995; see also Hubeny 1990). We write:

$$
q_{\nu}^{-}=\sum_{i} \frac{(7 / 8) \sigma T^{4}}{(3 / 4)\left(\tau_{\nu_{i}} / 2+1 / \sqrt{3}+1 / 3 \tau_{a, \nu_{i}}\right)} .
$$

Here $\tau_{\nu_{i}}=\tau_{a, \nu_{i}}+\tau_{s, \nu_{i}}$ is the sum of absorptive and scattering optical depths calculated for each neutrino flavor and $\tau_{a, \nu_{i}}$ the total absorptive optical depth for each neutrino flavor (e.g. for $\nu_{e}$, the absorptive optical depth includes all terms, $\tau_{a, \nu_{e}}=\tau_{a, \nu_{e} \bar{\nu}_{e}}+\tau_{a, e N}+\tau_{a \text {,brem }}+\tau_{a \text {,plasmon }}$ and the last two terms are negligible). Equation (15) gives the correct behavior in the limit of both small and large $\tau_{\nu_{i}}$ and $\tau_{a, \nu_{i}}$.

For simplicity, we approximate $q_{\text {adv }}$ by, (see e.g., Narayan \& Yi 1994; Abramowicz et al. 1995):

$$
q_{\mathrm{adv}}=\Sigma v T \frac{d s}{d r} \simeq \xi v \frac{H}{R} T\left(\frac{11}{3} a T^{3}+\frac{3}{2} \frac{\rho k}{m_{p}} \frac{1+X_{\mathrm{nuc}}}{4}\right),
$$

where $\xi \propto(-d \ln s / d \ln r)$ (see e.g.; Kato, Fukue \& Mineshige 1999 for the exact expression for $\xi)$ is taken to be constant and equal to 1 . The factor $(11 / 3) a T^{3}$ is the entropy density of the radiation. The entropy density of neutrinos, a factor $(7 / 6) a T^{4}=4 / 3 \times 7 / 8 a T^{4}$, is also added to equation (16) when the corresponding pressure term is added to the equation of state. Note that degeneracy pressure makes no contribution to the advection term because there is no associated entropy.

We solve numerically equations (13)-(16) to find the temperature and density at a given radius and for a given $\dot{m}$. We are interested primarily in the properties of the inner regions of the accretion flow, where neutrino processes are important. We thus concentrate on the range of radii from $r_{\min }=3$ to $r_{\max }=200$. (From the work of PWF and NPK we know that, for $r \gtrsim 100$, the flows are fully advection dominated since neutrino cooling is not important and photons are completely trapped).

\footnotetext{
${ }^{8}$ Equation (15) was derived in the context of radiative transport and assumes that opacities and emissivities are independent of the $z$ coordinate. Although we do not solve for the vertical structure of the flow, this assumption is less accurate for the neutrino transport where all cross sections are a function of temperature and hence of the vertical disk structure
} 


\section{Numerical results}

\subsection{Gas profiles, neutrino optical depths and advection}

Temperature and density profiles calculated from our model are shown in Figure 1 and the corresponding pressure components in Figure 2. We show our solutions for three values of the accretion rate, $\dot{m}=0.1,1,10$ (long dashed, solid and short dashed line, respectively). The thinner lines show the corresponding PWF solutions (see also their Figure 3). For easier comparison between the two sets of solutions, we extend our solution range out to $r=1000$. PWF and NPK have shown that, at large radii, densities and temperatures are too small for neutrino cooling to be significant, while optical depths are too large for photon cooling so that the flows are simply advection dominated. Therefore the solutions should not be sensitive to any assumption made about neutrino cooling and should compare well in this region. Figure 1 shows that, at large radii, there is indeed good agreement, both in the slope and in the normalization, between our solutions and those of PWF. In this region, radiation pressure dominates for $\dot{m}<10$, whereas the degeneracy and gas pressure components become increasingly more dominant for the higher values of $\dot{m}$ (Figure 2). In the region from $r \sim 100$ to $\sim 300$, the temperature profiles flatten. Here, photodisintegration and, more importantly, neutrino emission starts cooling the gas. The treatment of neutrino transfer inside this region becomes important and the two solutions start differing. Note that the onset of photodisintegration produces a sharp feature in the PWF solution in this range of radii. Such a feature is also reproduced in our calculation although it is somewhat weaker, particularly as seen in the density profiles.

Inside radii of $r \sim 30-100$, and for $\dot{m} \gtrsim 1$, our temperature solution steepens again whereas the PWF profile flattens. A similar trend is observed in the density profiles. In this region gas pressure becomes the dominant term in the equation of state (Figure 2).

Figure 3 shows contour plots for three quantities: the optical depths $\tau_{a, \nu}=\sum \tau_{a, \nu_{i}}$ (dotted lines) and $\tau_{s, \nu}=\sum \tau_{s, \nu_{i}}$ (dashed line) in the top panel, the advection parameter, $f=q_{a d v} / q^{+}=1-q^{-} / q^{+}$in the middle panel and the viscous timescale, $t_{\text {acc }}=R^{2} / \nu=\alpha^{-1}(H / R)^{-2} \Omega_{K}^{-1}$ in the bottom panel. These quantities have been calculated for $\dot{m}$ ranging from 0.1 to 10 in a region of the disk from $r=3$ to 200 (where neutrino cooling is important). The top panel of Figure 3 shows that the optical depth is dominated by the absorptive opacity in the inner regions of the flow (and in particular by the inverse of proton and neutron capture, given by Eq. 4). The optical depth exceeds 1 for $r$ in the range $\lesssim 5-30$ and $\dot{m}=0.1-10$. Note that the cooling function in equation (15) reduces to the optically thin expression for small optical depths but it differs significantly from the latter at optical depths $\sim 1$ and larger. The

treatment of neutrino transport via equation (15), in the optically thick regions, is what gives rise to the major differences between our work and that of PWF.

The most significant consequence of the gas becoming opaque is that neutrinos are trapped in the inner regions of the flow and the neutrino emission is partially suppressed. This is illustrated 
by the middle panel in Figure 3. Energy advection becomes important (i.e. $f>0.5$ : advection dominates over cooling) in the inner region of the flows and in particular in the region where the optical depths become significantly larger than 1. By comparing the contours of the optical depths and $f$ one finds that the region where most of the neutrino cooling occurs (where $f$ decreases) lies roughly along (or just outside) the $\tau=1$ contours. With increasing $\dot{m}$ the neutrino cooling becomes increasingly less efficient as the $\tau=1$ surface, moves further out in the flow. Consistently with previous work, we also find that $f$ (i.e. advection) also dominates at large radii. In particular, $f \sim 1$ for $r \gtrsim 100$; this is equivalent to the statement that the cooling timescale is much longer than the accretion timescales, so the energy is advected inward before it can be radiated away.

In our solution, which is mostly advection-dominated, we have $H \sim R$. Therefore, $t_{\text {acc }}$ is almost independent of $\dot{m}$ and approaches the simple dependence: $t_{\text {acc }} \sim 1 / \alpha \Omega_{K}$. Our values of $t_{\text {acc }}$ are typically a factor $\sim 10$ smaller than in Figure 1 of NPK, where the advection term and the appropriate opacities were not taken into account self-consistently within the NDAF region.

In the optically thin approximation used by PWF, the neutrino emission increases with increasing $\dot{m}$ and energy advection is not important in the inner regions of the flow. Although PWF also estimated that the flows become optically-thick to their neutrino emission above $\dot{m} \sim 1$ (consistent with our results), they did not account for neutrino opacities in their models. Our results, although based on a simple model, emphasize the importance of accounting for neutrino transfer'? We find that, for most of the range of accretion rates of interest for GRB models, a neutrino-dominated accretion flow becomes optically thick to neutrinos, and advection of energy provides the most significant cooling term in its central regions. In $\S 5$, we will discuss the implications of these results for the accretion flow neutrino luminosities in GRBs.

\section{Stability}

NPK discussed the stability properties of their NDAF solution. Since our model differs considerably from theirs, we repeat the analysis here.

The general condition for thermal stability is:

$$
\left(\frac{d \ln Q^{+}}{d \ln T}\right)_{\mid \Sigma}<\left(\frac{d \ln Q^{-}}{d \ln T}\right)_{\mid \Sigma},
$$

where $Q^{-}=q^{-}+q_{\text {adv }}$. The top panel of Figure 4 shows the two functions $Q^{+}$and $Q^{-}$(solid and dashed line respectively) as a function of the temperature of the gas. The radius is fixed at

\footnotetext{
${ }^{9}$ We note that PWF considered a fully-relativistic Kerr geometry. Our solution does not take into account the effects of black hole rotation. However the differences between our solution and that of PWF are always much larger than those introduced by allowing for black hole rotation (see e.g.; Figure 6 in PWF). Although the black hole is most likely rotating, we do not consider our main conclusions to be significantly limited by adopting the simple Newtonian treatment. This is because, as shown in Figure 3, most of the neutrino energy comes from radii of several $R_{\mathrm{S}}$.
} 
$r=100$ (for the lower set of curves) and $r=10$ (for the upper set of curves which have also been renormalized by a factor $10^{5}$ ), while the surface density is taken to be $\Sigma=10^{16} \mathrm{~g} \mathrm{~cm}^{-3}$. The equilibrium value of $T$ is given by the intersection of the two curves. We see that the stability condition above is satisfied at this point, so that the solution is thermally stable.

The condition for viscous stability is given by

$$
\frac{d \dot{m}}{d \Sigma}>0
$$

The bottom panel of Figure 4 shows $\dot{m}$ versus $\Sigma$ at $r=100$ (solid line) and at $r=10$ (dotted line). This shows that the disk is viscously stable. We have checked both the thermal and viscous stability conditions for a variety of $r$ and $\dot{m}$ and we find that the flows are stable under all conditions. This is a well known property of flows in which advection is important.

Finally, we also check for the gravitational stability condition, namely that the Toomre parameter $Q_{T}$ should be larger than 1 . For a Keplerian disk, $Q_{T}$ is given by:

$$
Q_{T}=\frac{c_{s} \kappa}{\pi G \Sigma}=\frac{\Omega_{K}^{2}}{\pi G \rho}
$$

where $\kappa$ is the epicyclic frequency. Figure 5 shows the variation of $Q_{T}$ with radius for $\dot{m}=0.1,1,3,10$. We see that $Q_{T}$ decreases with increasing $r$, so that the flows are most unstable on the outside (as found also by NPK). However, only for the largest $\dot{m} \sim 10$ and for $r \gtrsim 50$ does $Q_{T}$ go below unity, signifying gravitational instability. Thus the flows are gravitationally stable under almost all conditions of interest.

\section{Discussion}

The relevance of accretion processes in the central engine of GRBs was highlighted by Narayan et al. (1992). PWF carried out the first detailed study of accretion around black holes with ultra-high $\dot{m}$ (of order a solar mass per second), and identified a new mode of accretion which they named neutrino-dominated accretion flow (NDAF). In a subsequent study, NPK noted that accretion flows with such high $\dot{m}$ are highly advection dominated at large radii $(r \gtrsim 100)$. They suggested that the flow at these outer radii may take the form of a convection-dominated accretion flow (CDAF; Narayan, Igumenshchev \& Abramowicz 2000; Quataert \& Gruzinov 2000) or a related kind of flow (e.g., Blandford \& Begelman 1999). As a result, only a small fraction of the available mass accretes on the black hole, the bulk of the gas being ejected from the system. Close to the black hole $(r \lesssim 100)$, however, NPK confirmed PWF's result, that a cooling-dominated NDAF should be present.

PWF and NPK made the simple assumption that the accreting gas is optically thin to its own neutrino emission. We have improved on this by including the effects of neutrino transfer (via a simple prescription) and the neutrino opacities self-consistently in the model. With these 
improvements, we find that the central regions of NDAFs are typically opaque, so that the neutrinos emitted by the accreting gas are largely trapped. The optically-thick region extends out to $r \sim 4-5$ at $\dot{m} \sim 0.1$ to $r \sim 30-40$ at $\dot{m} \sim 10$ (Figure 3 ). We have shown that, above $\dot{m} \gtrsim 1$, the majority of the energy liberated by viscous dissipation is advected with the flow instead of being emitted in the form of neutrinos, and energy advection is much more important in the inner regions of the accretion flows than previously realized (Figure 3).

In order to further explore the implications of these results we now calculate the neutrino luminosity from our model and the fraction of the binding energy carried out to infinity by neutrinos. We also derive the neutrino Eddington luminosity for such flows.

Figure 6 shows the neutrino luminosity from the accretion flow, $L_{\nu}=\int_{r_{\min }}^{r_{\max }} 2 \pi q_{\nu}^{-} r d r$, (in units of $10^{51} \mathrm{erg} \mathrm{s}^{-1}$; solid line; upper panel). Also, using the opacities discussed in $\S 2.2$ we derive the neutrino Eddington luminosity of the flow:

$$
L_{\mathrm{Edd}, \nu}=\frac{4 \pi G M c}{\kappa_{\nu}} \sim 9 \times 10^{53} m_{3} T_{11}^{-2} \operatorname{erg~s}^{-1},
$$

where, for illustration the numerical value given on the right uses only the two dominant components of the opacity: neutron and proton absorption and scattering (for electron neutrinos this gives $\kappa_{\nu_{e}}=\kappa_{\nu_{e}, a}+\kappa_{\nu_{e}, s}=5.5 \times 10^{-17} T_{11}^{2} \mathrm{~cm}^{2} \mathrm{~g}^{-1}$; Equations 8 and 11 ; note that for the calculation shown in Figure 6 all opacity terms are used). Because the neutrino cross sections are a function of neutrino energies, the neutrino Eddington luminosity is a function of both black hole mass and gas temperature. In Figure 6, we show the neutrino Eddington luminosity calculated for $T_{11}=T_{11}(r=3)$, the temperature at the inner radius of the flow; at larger radii the Eddington luminosity increases. In the middle panel of Figure 6 we also show the neutrino radiative efficiency, defined, as in standard accretion theory, by $\eta_{\nu}=L_{\nu} / \dot{M} c^{2}$.

The neutrino luminosity from the flow is $L_{\nu} \sim 10^{51} \mathrm{erg} \mathrm{s}^{-1}$ at $\dot{m} \sim 0.01$ and increases almost linearly with $\dot{m}$ up to $\dot{m} \sim 0.1$ (Figure 6 ). Between $0.1 \lesssim \dot{m} \lesssim 1$, the luminosity flattens off significantly, ranging within $6-8 \times 10^{52} \mathrm{erg} \mathrm{s}^{-1}$. Above $\dot{m} \sim 1$ the neutrino luminosity stays virtually constant at $L_{\nu} \sim 8 \times 10^{52} \mathrm{erg} \mathrm{s}^{-1}$. In contrast, in the PWF solution, which assumes optically thin neutrino emission, the neutrino luminosity ranges from $L_{\nu} \sim 10^{51-52} \mathrm{erg} \mathrm{s}^{-1}$ for $\dot{m}=0.1$ to up to $L_{\nu} \sim 10^{54} \mathrm{ergs}^{-1}$ for $\dot{m}=10$ (see their Table 3). Thus, one important consequence of the addition of neutrino opacities is that the flow luminosity varies only by a factor of a few over the range of accretion rates appropriate for popular models of GRB progenitors. The luminosity of the flow is almost constant because the efficiency with which energy is transported out of the flow by neutrinos, $\eta_{\nu}$ (bottom panel, Figure 6) decreases with increasing $\dot{m}$ (or equivalently, as shown in Figure 5, decreases as neutrinos become trapped and energy advection becomes more dominant at larger $\dot{m}$ ). We show that $\eta \sim 0.1$ (consistent with the efficiency expected from a geometrically thin, cooling dominated Newtonian accretion disk) up to $\dot{m} \sim 0.1$ and decreases (almost linearly) with increasing $\dot{m}$ (reaching $\eta \sim$ a few $10^{-3}$ at $\dot{m}=10$ ). The fraction of energy transported away by neutrinos decreases as the inner regions of the flow become neutrino opaque (see Figure 3). 
Figure 6 also shows the neutrino Eddington luminosity evaluated at the inner edge of the disk (dashed line). In the inner regions of the flow, the temperature is the highest, and consequently the Eddington luminosity the lowest (Eq. 20), so that $L_{\nu} \sim L_{\text {Edd }, \nu}$ for $\dot{m} \gtrsim 1$. Momentum deposition in the inner regions of the flow by neutrinos may therefore be quite effective as a mechanism responsible for ejection.

We now examine the implications of our results for GRBs. A number of authors, including Eichler et al. (1989; see also Narayan et al. 1992) suggested that $\nu \bar{\nu}$ annihilation around merging compact binaries might produce a relativistic $e^{+} e^{-}$fireball with sufficient energy to power a GRB. Detailed numerical simulations by Janka et al. (1999) show that this process might conceivably power short GRBs (those with durations under a couple of seconds or so), provided that there is a modest level of beaming. However, we have shown that neutrino advection is important in most of the parameter space of these models, which may have a significant impact on the efficiency of this process. We estimate the luminosity due to $\nu \bar{\nu}$ annihilation along $z$-axis above the disk (using an improved version of Eq. 10 in Ruffert et al. (1997) provided by Thomas H. Janka, private communication) to be:

$$
\mathrm{E}_{\nu \bar{\nu}} \sim 6 \times 10^{-35} \frac{2\left\langle E_{\nu}\right\rangle L_{\nu}^{2}}{\pi c^{2}\left[1-\left(R_{\min } / R_{\text {surf }}\right)^{2}\right]^{2}} \frac{1}{R_{\text {surf }}} \int_{0}^{\infty} d \epsilon \Phi\left(\cos \theta_{\min }-\cos \theta_{\text {surf }}\right)^{2} \operatorname{erg~s}^{-1},
$$

where the constant in front takes into account the neutrino cross sections. $L_{\nu}$ is given above and shown in Figure 6 and $R_{\text {surf }}$ and $\left\langle E_{\nu}\right\rangle$ are evaluated at the neutrinosphere, defined as the surface at which the emergent neutrinos originate. Hence, $\left\langle E_{\nu}\right\rangle$ is the average energy of the escaping neutrinos given by $\left\langle E_{\nu}\right\rangle=3.7 k T_{11 \text {,surf }}$ (Eq. 10) with $T_{11 \text {, surf }}=T_{11}\left(r_{\text {surf }}=r\left(\tau_{\nu}=2 / 3\right)\right.$ ) and $\tau_{\nu}=\tau_{a, \nu}+\tau_{s, \nu}$. If the spectrum is a blackbody, the radius of this surface is located near and above the layer of optical depth $=2 / 3$ (this is because the opacity is mostly dominated by absorption processes). The contour of $\tau_{\nu}=2 / 3$ and the radius of the neutrinosphere as a function of $\dot{m}$ is shown in Figure 3. We find that the radius of the neutrinosphere 10 to be given by, $r_{\text {surf }}=r_{\tau=2 / 3} \sim 17 \dot{m}^{2 / 5}$ in the range $0.1 \lesssim \dot{m} \lesssim 10$. At this radius the temperature of the neutrinosphere is roughly $T_{11 \text {,surf }} \sim 0.2 \dot{m}^{-3 / 20}$ which is fairly insensitive to $\dot{m}$ in the range from $0.1 \lesssim \dot{m} \lesssim 10$ ). The integral part in equation (21) is a geometrical factor, where $\Phi=3 / 4\left[1-2\langle\mu\rangle^{2}+\left\langle\mu^{2}\right\rangle^{2}+1 / 2\left(1-\left\langle\mu^{2}\right\rangle\right)^{2}\right]$, where $\left\langle\mu^{n}\right\rangle=\int_{\mu_{\text {surf }}}^{\mu_{\min }} d \mu \mu^{n} / \int_{\mu_{\text {surf }}}^{\mu_{\min }} d \mu$ with $\mu_{\text {surf }}=\cos \theta_{\text {surf }}=1 / \sqrt{1+\left(r_{\text {surf }}^{2} / z^{2}\right)}, \mu_{\min }=\cos \theta_{\min }=1 / \sqrt{1+\left(r_{\min }^{2} / z^{2}\right)}$ and $\epsilon=z / r_{\text {surf }}$.

The bottom panel of Figure 6 shows the neutrino annihilation luminosity, $L_{\nu \bar{\nu}}$ in units of $10^{51} \mathrm{erg} \mathrm{s}^{-1} . L_{\nu \bar{\nu}}$ increases up to its maximum value of $\sim 10^{50} \mathrm{erg} \mathrm{s}^{-1}$ at $\dot{m} \gtrsim 1$ and slightly decreases for larger $\dot{m}$. Our estimate of $L_{\nu, \bar{\nu}}$ at $\dot{m}=1$ agrees well with values estimated in the simulations of Ruffert \& Janka (1999) and PWF (although our results are less accurate than those of PWF for $\dot{m} \lesssim 0.1$ ). In accordance with PWF we find that energetic events can only be achieved

\footnotetext{
${ }^{10}$ To derive the analytical scalings we solve Eqs. (13) and (14) assuming $P=P_{\text {gas }}$ and $q^{+}=q_{a d v}^{-}$. We have shown that is a good approximation within the optically thick region.
} 
for $\dot{m}>0.1$ (below which the neutrino annihilation efficiency decreases very sharply; see also their Table 3); but in contrast with their results we do not find that increasingly more energetic events can be achieved for larger accretion rates. Our calculations imply that the efficiency of $\nu \bar{\nu}$ annihilation remains constant (or slightly decreases) for $\dot{m} \gtrsim 1$. This is expected, as we have found that neutrinos are increasingly more trapped in the disk. (Our estimate may be uncertain by a factor $\sim 2$; this is mostly due to the fact that our solution gives only height-averaged quantities whereas the vertical disk stratification may be important in this calculation). Note also that recent results from hydrodynamical calculations carried out by Lee \& Ramirez-Ruiz (2002) show that $\nu \bar{\nu}$ annihilation can only produce bursts from impulsive energy inputs, as the annihilation luminosity scales as $t^{-5 / 2}$. This further restricts the importance of this process to a small fraction of bursts.

Energy extraction from the disk is also possible by MHD processes. Such processes are broadly based on the expectation that the differential rotation of the disk will amplify pre-existing magnetic fields, until they approach equipartition with the gas kinetic energy. Proposed mechanisms include Parker instabilities in the disk leading to reconnection, relativistic flares and winds (Narayan et al. 1992; Meszaros \& Rees 1997) or the Blandford-Znajek mechanism (Blandford \& Znajek 1977; hereafter BZ). Of these, perhaps, the Blandford-Znajek efficiency is the easiest to estimate (at least roughly). We follow the common assumption that the magnetic field in the disk will rise to some fraction of its equipartition value $B^{2} / 8 \pi \sim \rho c_{s}^{2}$. Typical values of $\rho c_{s}^{2}$ are $10^{30-32} \mathrm{erg} \mathrm{cm}^{-3}$, for $0.1<\dot{m}<10$ (see Figure 2), implying a field strength of $\sim 10^{15-16} \mathrm{G}$ if we make the conservative assumption that $B$ is only $10 \%$ of its equipartition value. Consistent with earlier work (PWF; Ruffert \& Janka 1999), the BZ jet luminosity at $\dot{m}=10$ is then

$$
L_{B Z}=\left(\frac{B^{2}}{4 \pi}\right) \pi R_{h}^{2} a^{2} c \gtrsim 10^{52} a^{2}\left(\frac{B}{10^{16} \mathrm{G}}\right)^{2}\left(\frac{M}{3 \mathrm{M}_{\odot}}\right)^{2} \quad \operatorname{erg~s}^{-1},
$$

where $R_{h}=2 G M / c^{2}$ is the black hole radius and $a=R_{h} \Omega_{h} / c$ is the dimensionless black hole spin parameter $(0<a<1) . L_{B Z}$ is about 2 orders of magnitude larger than the neutrino annihilation rate, $L_{\nu \bar{\nu}}$, at $\dot{m} \sim 10$. For larger equipartition fractions, the ratio $L_{B Z} / L_{\nu \bar{\nu}}$ becomes large even at $\dot{m} \sim 0.1$ or 1 . It is obvious that the energy liberated by this mechanism is simply proportional to the internal energy density of the disk and hence is not affected by advection. Indeed, Livio, Ogilvie \& Pringle (1999) have argued that the BZ mechanism might be most relevant for advection dominated flows (see also Meier 2001). Numerical simulations such as those by MacFadyen \& Woosley (1999) and MacFadyen, Woosley \& Heger (2001) have shown that the BZ mechanism would be an efficient mechanism capable of liberating a large fraction of the black hole spin energy.

By similar electromagnetic considerations as those used to derive the BZ luminosity, Livio et al.(1999) estimate that the electromagnetic/wind power output from a disk is given by:

$$
L_{d}=\left(\frac{B_{p d}^{2}}{4 \pi}\right) \pi R_{d}^{2}\left(\frac{R_{d} \Omega_{d}}{c}\right) c \sim\left(\frac{B_{p d}}{B}\right)^{2}\left(\frac{R_{d}}{R_{h}}\right)^{3 / 2} a^{-2} L_{B Z} \sim 5 a^{-2} L_{B Z}
$$

where, following Livio et al., we take $R_{d} \Omega_{d} / c \sim\left(R_{h} / R_{d}\right)^{1 / 2} . B_{p d}$ is the poloidal field in the disk given, approximately, by $B_{p d} \sim(H / R) B$ (see also Merloni \& Fabian 2002). Because $H / R \sim 1$ in 
the inner region of the disk we have $B_{p d} \sim B . R_{d}$ is a factor of a few times $R_{h}$ (above we take $R_{d}$ as the radius of the innermost stable orbit, at which the disk energy density is the highest). Since $a^{2}<1$ and, $R_{d}$ is at least a few times $R_{h}$, it is evident that in a geometrically thick disk, the electromagnetic/wind luminosity from the disk can easily be larger than the BZ luminosity, hence $L_{d}>>L_{\nu \bar{\nu}}$. Our calculations therefore indicate that, with increasing accretion rates, MHD processes become significantly more efficient at releasing energy than neutrino annihilation processes, hence they are probably the most viable mechanisms for energy extraction in these systems.

Although the stability analysis presented in $§ 4$ shows that the accretion flows we have studied are intrinsically stable, we note that the viscous time scale (Fig. 3) can be as short as a small fraction of a second. Therefore, if the accretion disk is fed in a variable manner, e.g., via fallback material after a supernova explosion as in the collapsar model, we may expect variations in the accretion rate. This might explain the complex light curves of GRB in at least some of the scenarios discussed above (e.g., MacFadyen \& Woosley, 1999).

We thank Thomas H. Janka for valuable comments and important suggestions. We also thank Chris Fryer and Stan Woosley for sending us the files of their temperature and density solutions used in Figure 1 and Andrea Merloni for discussion. T.D.M. aknowledges support by grant NAG-10105. R. P. acknowledges support by the IAS-CNR (Rome, Italy) during the time that part of this research was conducted. R. N. was supported in part by the W. M. Keck Foundation as a Keck Visiting Professor at the Institute for Advanced Study. R. N.'s research was supported in part by NSF grant AST-9820686.

\section{REFERENCES}

Abramowicz, M., Czerny, B., Lasota, J.P., \& Szuszkiewicz, E., 1988, ApJ, 332, 646

Abramowicz, M. A., Igumenshchev, I. V., Quataert, E., \& Narayan, R. 2001, ApJ, in press astro-ph/0110371)

Begelman, M.C., 1978, MNRAS, 184, 53

Blandford R.D., \& Begelman M.C., 1999, MNRAS, 303, L1

Blandford, R.D. \& Znajek, R.L., 1977, MNRAS, 179, 433

Bethe H.A., Applegate J.H., \& Brown G.E., 1980, ApJ, 241, 343

Eichler, D., Livio, M., Piran, T., \& Schramm, D.N., 1989, Nature, 340, 126

Frail, D.A., et al. 2001, ApJ, 562, L55

Fryer, C.L., \& Woosley, S.E., 1998, ApJ, 502, L9

Hannestad S., Raffelt, G., 1998, ApJ, 507,339 
Hubeny, I., 1990, ApJ, 351, 632

Itoh, N., Adachi, T., Nakagawa, M., Kohyama, Y., \& Munakata H., 1989, ApJ, 339, 354

Itoh, N., Adachi, T., Nakagawa, M., Kohyama, Y., \& Munakata H., 1990, ApJ, 360, 741

Janka, H. T., Eberl, T., Ruffert, M., \& Fryer, C. L. 1999, ApJ, 527, L39

Kato S., Fukue J., Mineshige S., 1999, in 'Black Hole Accretion disks', eds by Kyoto University Press, Kyoto, Japan, p. 245

Katz, J., 1977, ApJ, 215, 265

Kohri K., Mineshige S., 2002, ApJ, submitted (astro-ph/0203177)

Igumenshchev, I. V., \& Abramowicz, M. A. 1999, MNRAS, 303, 309

Lee W.H., Ramirez-Ruiz E., 2002, ApJ, in press (astro-ph/0206011)

Livio M., Ogilvie G.I., Pringle J.E., 1999, ApJ, 512, 100

MacFadyen, A., \& Woosley, S.E., 1999, ApJ, 524, 262

MacFadyen, A., Woosley, S.E., \& Heger A., ApJ, 2001, 550, 410

Meier D.L., 2001, ApJ, 548, L9

Merloni A., Fabian A.C., 2002, MNRAS, 332, 165

Narayan, R., Igumenshchev, I. V., \& Abramowicz, M. A. 2000, ApJ, 539, 798

Narayan, R., Paczynski B., \& Piran, T., 1992, ApJ, 395, L83

Narayan, R., Piran, T., \& Kumar, P., 2001, ApJ, 557, 949

Paczynski, B., 1991, Acta Ast, 41, 257

Panaitescu, A., \& Kumar P., ApJ, 2001a, 554, 667

Panaitescu, A., \& Kumar P., ApJ, 2001b, 560, L49

Popham, R., \& Narayan, 1995, ApJ, 422, 337

Popham, R., Woosley, S.E., \& Fryer, C., 1999, ApJ, 518, 356

Quataert, E., \& Gruzinov, A. 2000, ApJ, 539, 809

Qian Y.Z., Woosley, S.E., 1996, ApJ, 471, 331

Ruffert M., Janka H.Th., Schäfer G., 1996, A\&A, 311,532

Ruffert M., Janka H.Th., 1999, A\&A, 344, 573

Ruffert M., Janka H.Th., 2001, A\&, 380, 544

Salpeter, E. E. \& Shapiro, S. L. 1981, ApJ, 251, 311

Shapiro, S.L., \& Teukolsky, S.A., 1983, in 'Black Holes, White Dwarfs and Neutron Stars', eds by John Wiley \& Sons, Inc; Chapter 18

Vietri, M. \& Stella, L. 1998, ApJ, 507, L45 
Usov, V.V., 1992, Nature, 357, 472

Woosley, S.E., 1993, ApJ, 405, 273

This preprint was prepared with the AAS $\mathrm{IAT}_{\mathrm{E}} \mathrm{X}$ macros v4.0. 
Fig.1 - Temperature and density profiles in the top and bottom panel respectively. The profiles are shown for three values of the accretion rate: $\dot{m}=10$ - short dashed lines; $\dot{m}=1$ - solid lines; $\dot{m}=0.1$ - long dashed lines. The PWF numerical solutions (thinner lines - see also their Fig. 3) are also plotted for direct comparison.

Fig. 2 - Pressure components for three values of $\dot{m}$. The gas pressure is shown by the solid line, degeneracy pressure by the dotted line, radiation pressure by the dashed line, neutrino pressure by the long dashed line

Fig.3 - Top panel: Contours showing the values of scattering optical depth $\left(\tau_{s}\right.$ - dashed line) and absorptive optical depth $\left(\tau_{a}\right.$ - dotted line) for a range of $\dot{m}=1-10$ and $r=3-200$. The $\tau=2 / 3$ contour is shown with the solid line. Middle panel: Contours showing the advection parameter $f=q_{a d v} / q^{+}$for the same range of $\dot{m}$ and as a function of $r$. For most regions of parameter space $f>0.5$ and advection dominates. Bottom panel: contours of the accretion time, $t_{a c c}$.

Fig.4 - Stability analysis. The top panel shows $Q^{+}$(dashed line) and $Q^{-}=q^{-}+q_{a d v}$ (solid line) versus $T$ for $r=100$ (bottom curves) and $r=10$ (top curves - renormalized by a factor of $10^{5}$ for better clarity) and for $\Sigma=10^{16} \mathrm{~g} \mathrm{~cm}^{-3}$. According to the criterion given in Equation 10, the flow is thermally stable. The lower panel shows $\dot{m}$ versus $\Sigma$ for $r=100$ (solid line) and $r=10$ (dotted line). The viscous stability condition (Eq. 11) is always satisfied.

Fig.5 - The Toomre parameter $Q_{T}$ (Eq. 12) as a function of radius for the four values of the accretion rate as in Fig. 2. $Q_{T}$ is always $>1$ (for $\dot{m} \gtrsim 3$ ) implying that the flow is gravitationally stable throughout and unstable in the outer regions for $\dot{m} \gtrsim 3$.

Fig.6 - Upper panel: the solid line represents the neutrino luminosity and the dashed lines the neutrino Eddington luminosity as a function of the accretion rate and for two values of the effective temperature. Middle panel: the solid line shows the radiative efficiency of the accretion flows as a function of $\dot{m}$. Bottom panel: $\mathrm{E}_{\nu \bar{\nu}}$ as a function of $\dot{m}$. The dotted line below $\dot{m}=0.1$ represents the most uncertain (upper limit) estimates. Better values of $\mathrm{E}_{\nu \bar{\nu}}$ for $\dot{m}<0.1$ are given in Table 3 of PWF. 

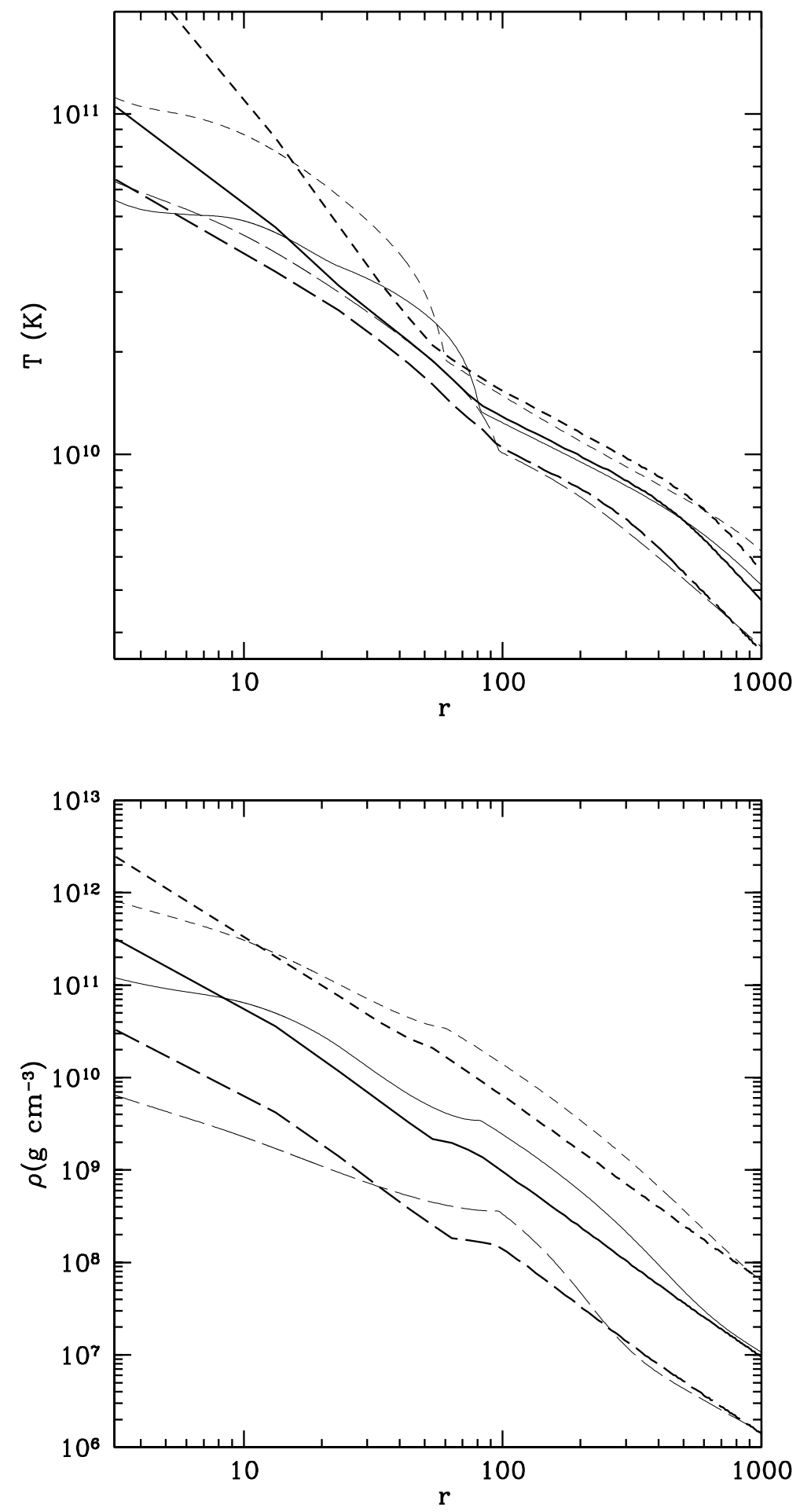


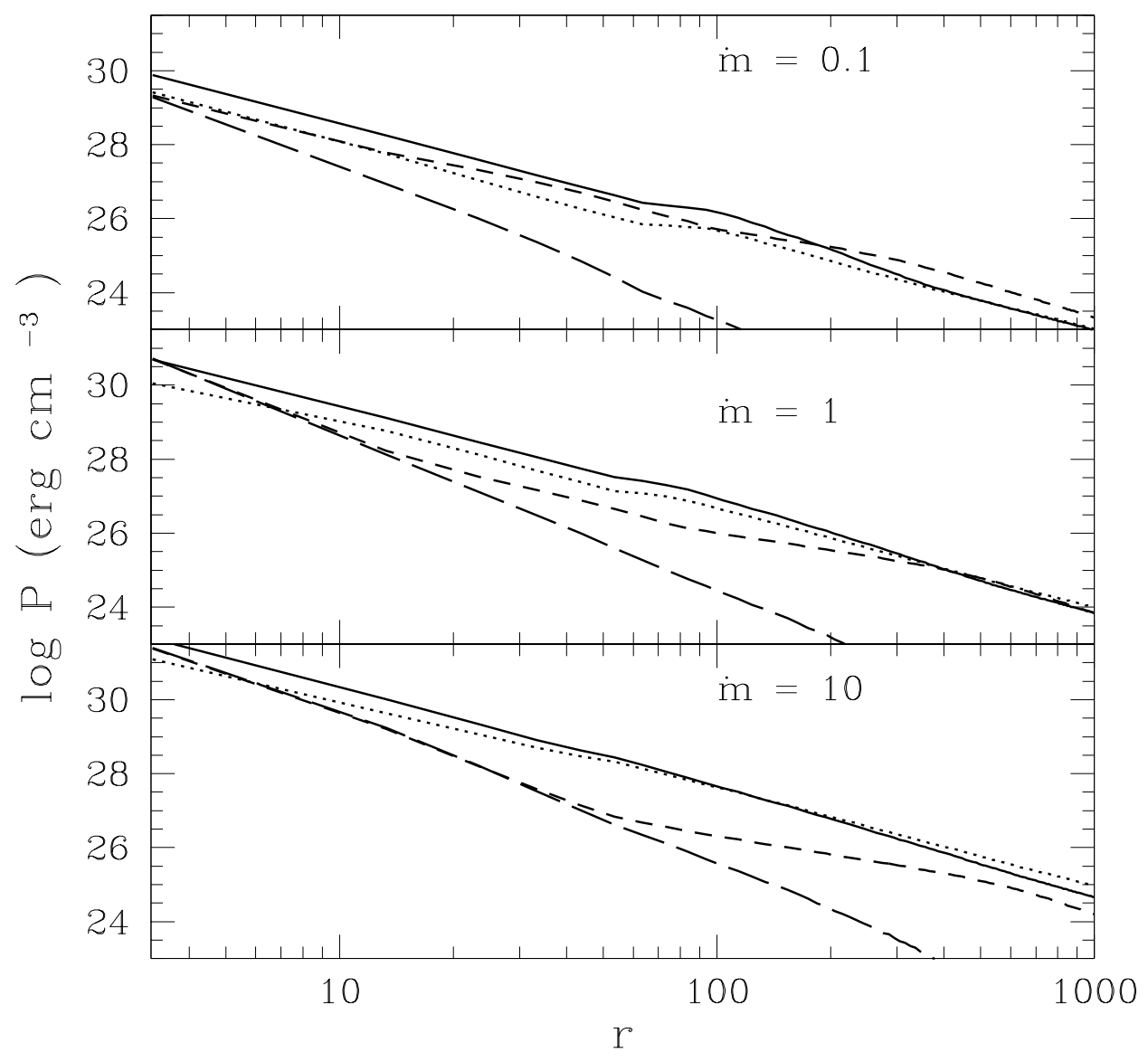



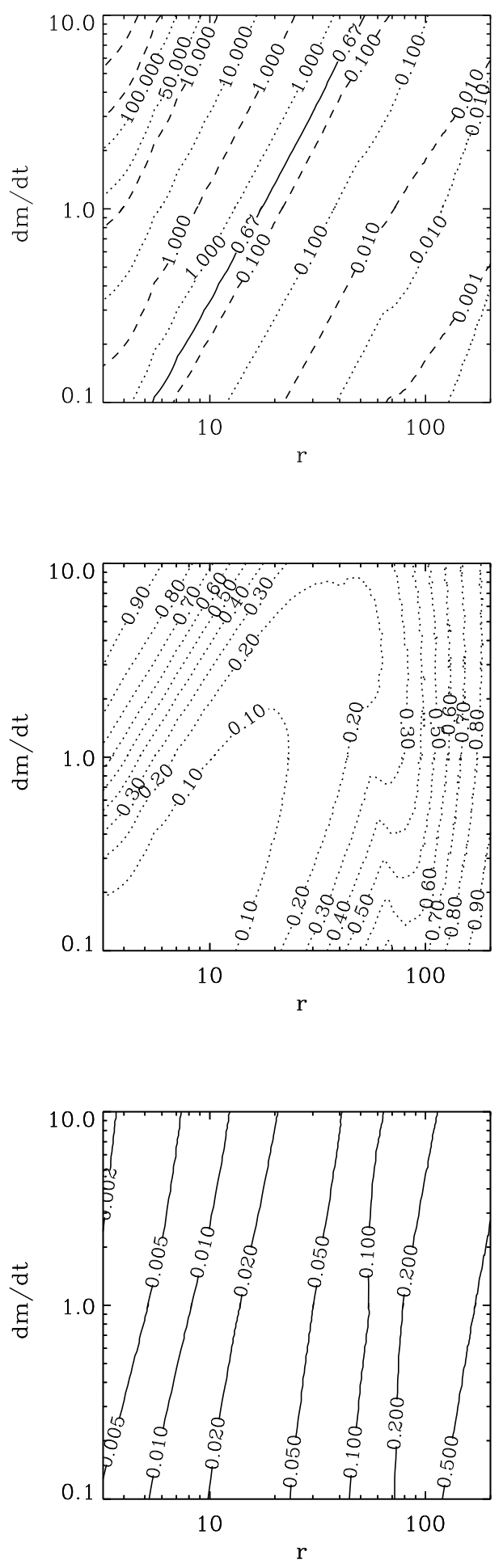

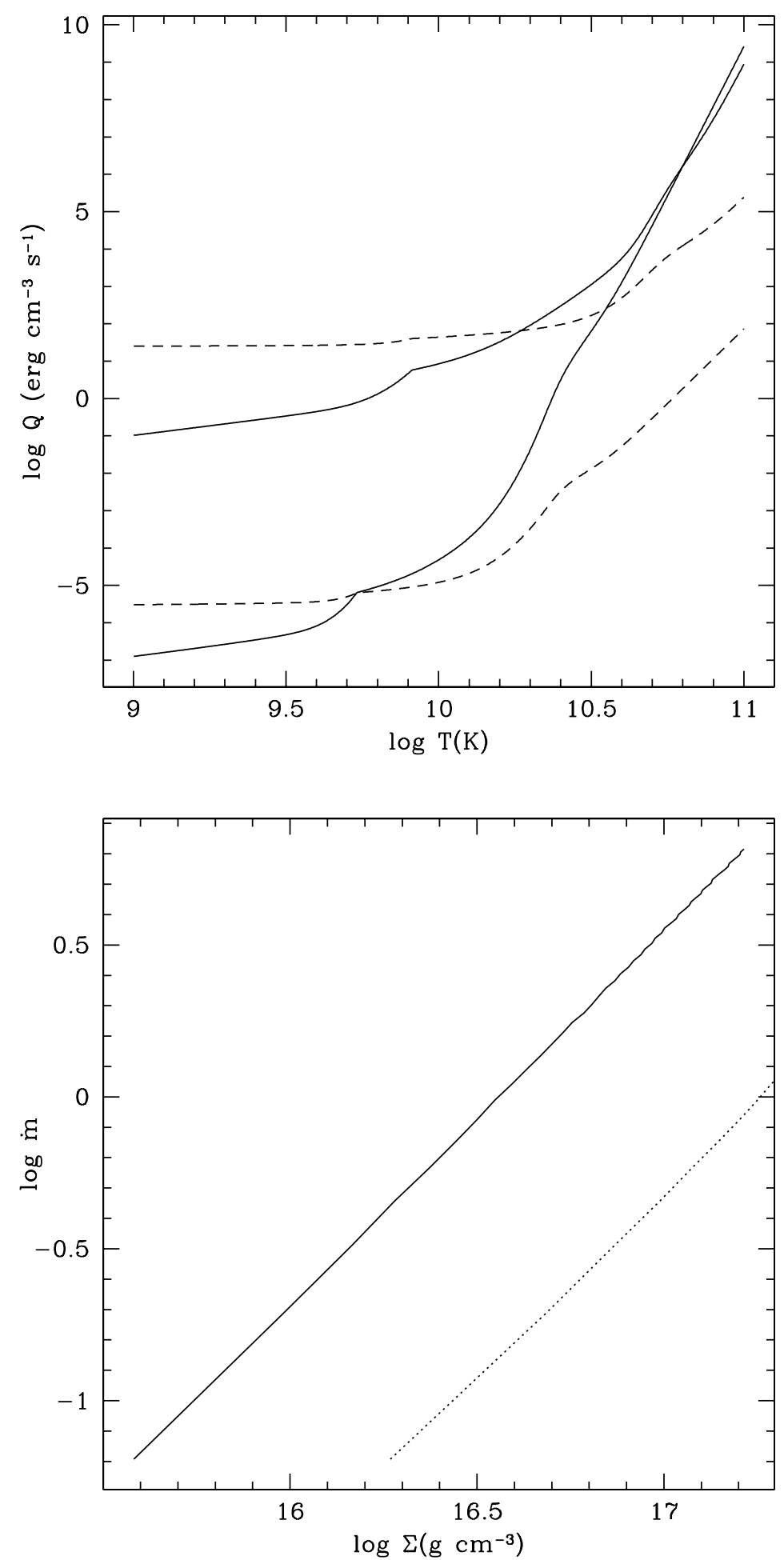


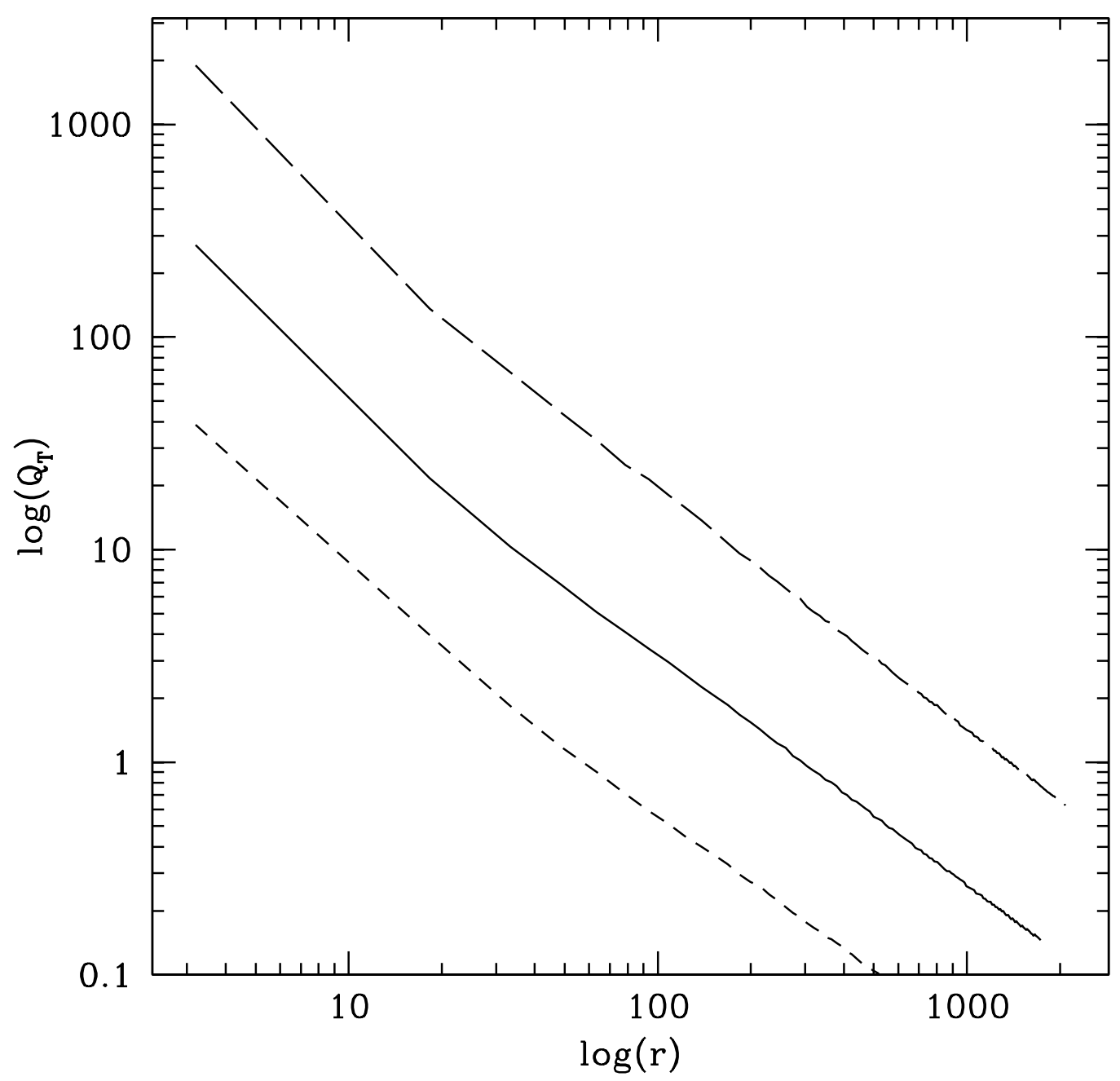



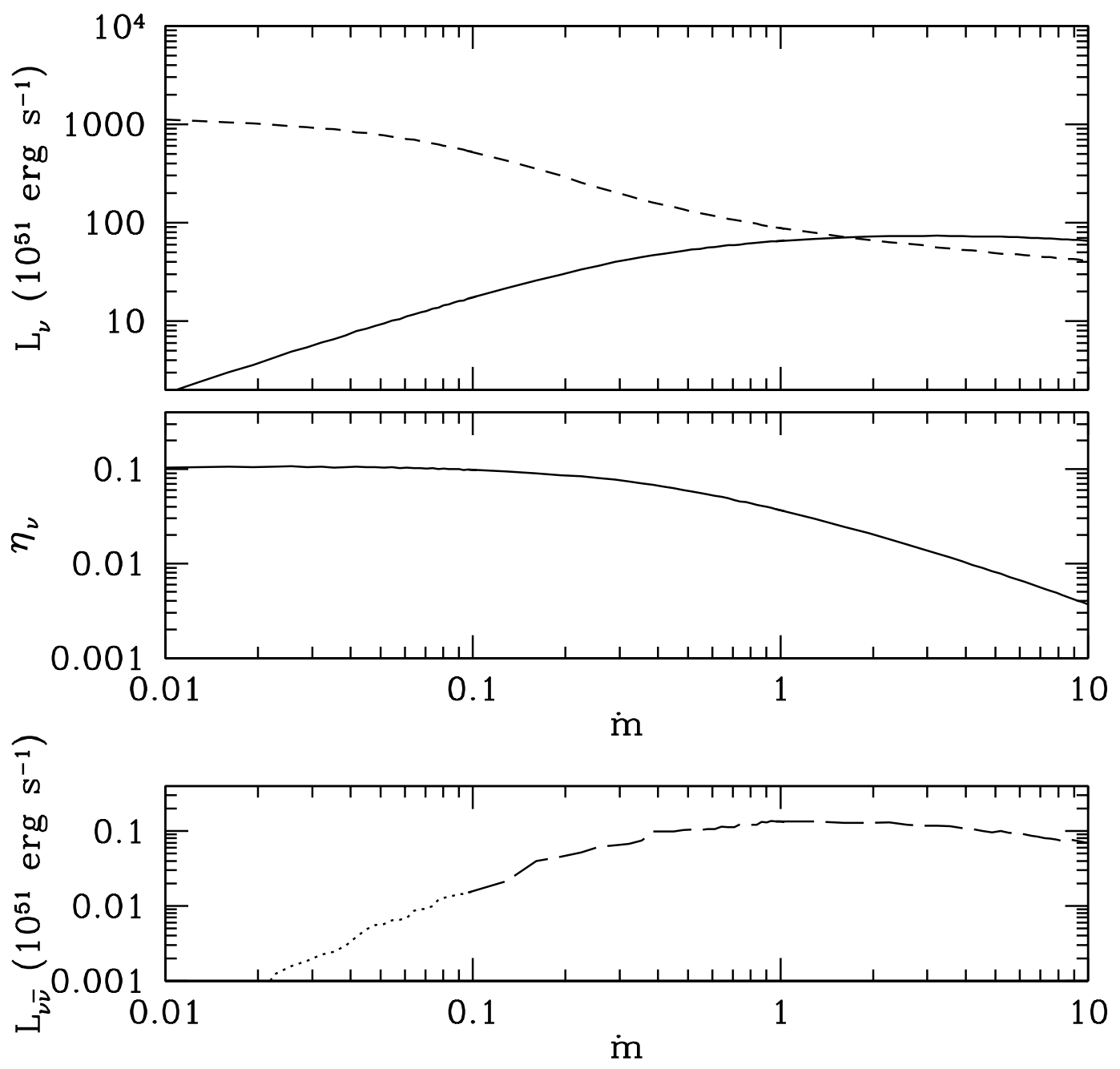